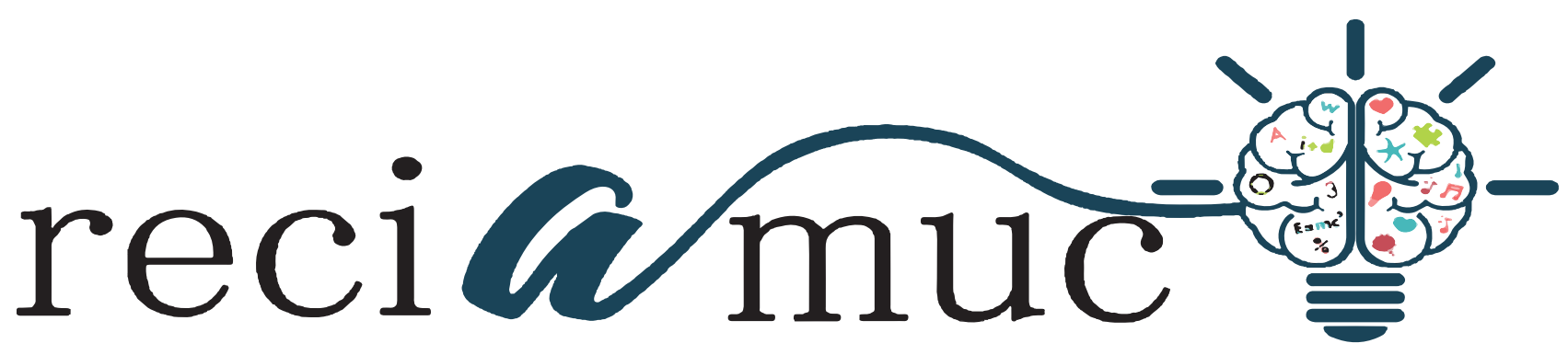

DOl: 10.26820/reciamuc/4.(4).diciembre.2020.14-21

URL: https://reciamuc.com/index.php/RECIAMUC/article/view/566

EDITORIAL: Saberes del Conocimiento

REVISTA: RECIAMUC

ISSN: 2588-0748

TIPO DE INVESTIGACIÓN: Artículo de Revisión

CÓDIGO UNESCO: 32 Ciencias Médicas

PAGINAS: $14-21$

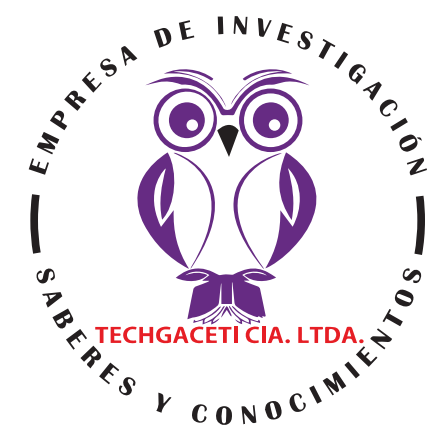

\title{
Trombólisis intraarterial en ACV isquémico agudo
}

Intra-arterial thrombolysis in acute ischemic stroke

Trombólise intra-arterial em acidente vascular encefálico isquêmico agudo

\section{María Dayana Velez Bravo'; Darío Iván Naranjo Apuntes²; Edwin Andrés Méndez Barbosa3; Jorge Adrián Barros Cabrera ${ }^{4}$}

RECIBIDO: 23/10/2020 ACEPTADO: 17/11/2020 PUBLICADO: 24/12/2020

1. Médico Cirujano; Médico General en Funciones Hospitalarias; Portoviejo, Ecuador; dayanavelezmaypa@hotmail.com (D) https://orcid.org/0000-0003-3370-0700

2. Medico; Investigador Independiente; Quito, Ecuador; darioivannaranjo@gmail.com (D) https://orcid.org/00000002-3302-002X

3. Médico y Cirujano General, Universidad Juan N. Corpas; Investigador Independiente; Colombia; menbar28@ hotmail.com (D) https://orcid.org/0000-0001-5499-9743

4. Médico General, Universidad de Cuenca; Investigador Independiente; Cuenca, Ecuador; jbc4444@hotmail. com (iD https://orcid.org/0000-0001-6720-732X

\section{CORRESPONDENCIA \\ María Dayana Velez Bravo \\ dayanavelezmaypa@hotmail.com \\ Guayaquil, Ecuador}

๑) RECIAMUC; Editorial Saberes del Conocimiento, 2020 


\title{
RESUMEN
}

El Accidente Cerebrovascular (ACV) isquémico es uno de los tipos más frecuentes de esta enfermedad cardiovascular (ECV). Representa entre un $80-85 \%$ de los de su tipo. El ACV isquémico es la tercera causa de muerte en los países desarrollados y la primera causa de discapacidad en adultos. La terapia trombolítica precoz en pacientes con ACV isquémico agudo reduce el área de infarto cerebral, además de mejorar los resultados funcionales a los 3 meses. Es fundamental para el clínico estar actualizado en cuanto a los avances terapéuticos que han demostrado impactar, en términos de calidad de vida y funcionalidad, en pacientes con esta patología. En consecuencia, el objetivo fundamental del presente estudio es plasmar los aspectos más resaltantes relacionados con la trombólisis intraarterial como tratamiento de pacientes con ACV isquémico agudo. El modelo de investigación es una revisión de tipo documental bibliográfico. La terapia trombolítica intraarterial en ACV isquémico agudo, conjuntamente o no, con la terapia IV es una gran alternativa, con excelentes resultados ampliamente comprobados. En virtud de lo cual, este tratamiento ha llegado a ser el estándar de asistencia en algunos centros importantes para ACV, mientras que, en muchos otros por razones técnicas o de falta de especialización, aún se encuentra ausente. La misión fundamental en el tratamiento del ACV isquémico agudo es impedir las pérdidas de tiempo en su aplicación con la finalidad de evitar los daños y complicaciones que pueda acarrear esta enfermedad. No obstante, este tipo de tratamiento, por su requerimiento de alta especialización, se encuentra reservado en muchos países a centros con alta tecnología.

Palabras clave: Trombólisis, Intraarterial, ACV, Isquémico, Agudo.

\begin{abstract}
Ischemic cerebrovascular accident (CVA) is one of the most frequent types of this cardiovascular disease (CVD). It represents between $80-85 \%$ of its kind. Ischemic stroke is the third leading cause of death in developed countries and the leading cause of disability in adults. Early thrombolytic therapy in patients with acute ischemic stroke reduces the area of cerebral infarction, in addition to improving functional results at 3 months. It is essential for the clinician to be updated regarding the therapeutic advances that have been shown to impact, in terms of quality of life and functionality, in patients with this pathology. Consequently, the main objective of this study is to capture the most salient aspects related to intra-arterial thrombolysis as a treatment for patients with acute ischemic stroke. The research model is a bibliographic documentary type review. Intra-arterial thrombolytic therapy in acute ischemic stroke, followed or not, with IV therapy is a great alternative, with excellent, widely proven results. As a result, this treatment has become the standard of care in some major stroke centers, while for many other technical reasons or lack of specialization, it is still absent. The fundamental mission in the treatment of acute ischemic stroke is to avoid wasting time in its application in order to avoid the damage and complications that this disease may cause. However, this type of treatment, due to its highly specialized requirement, is reserved in many countries for centers with high technology.
\end{abstract}

Keywords: Thrombolysis, Intraarterial, CVA, Ischemic, Acute.

\section{RESUMO}

O acidente vascular isquêmico cerebrovascular (AVC) é um dos tipos mais freqüentes desta doença cardiovascular (DCV). Ela representa entre 80 - 85\% de seu tipo. O AVC isquêmico é a terceira principal causa de morte nos países desenvolvidos e a principal causa de incapacidade em adultos. A terapia trombolítica precoce em pacientes com derrame isquêmico agudo reduz a área de infarto cerebral, além de melhorar os resultados funcionais aos 3 meses. É essencial que o clínico esteja atualizado em relação aos avanços terapêuticos que demonstraram ter impacto, em termos de qualidade de vida e funcionalidade, em pacientes com esta patologia. Consequentemente, o objetivo principal deste estudo é capturar os aspectos mais salientes relacionados à trombólise intra-arterial como tratamento para pacientes com derrame isquêmico agudo. O modelo de pesquisa é uma revisão do tipo documentário bibliográfico. A terapia trombolítica intra-arterial no acidente vascular cerebral isquêmico agudo, seguido ou não, com terapia intravenosa é uma ótima alternativa, com excelentes resultados amplamente comprovados. Como resultado, este tratamento tornou-se o padrão de tratamento em alguns grandes centros de AVC, enquanto que por muitas outras razões técnicas ou falta de especialização, ele ainda está ausente. A missão fundamental no tratamento do derrame isquêmico agudo é evitar a perda de tempo em sua aplicação a fim de evitar os danos e complicações que esta doença pode causar. Entretanto, este tipo de tratamento, devido a sua exigência altamente especializada, é reservado em muitos países para centros com alta tecnologia.

Palavras-chave: Thrombolysis, Intraarterial, CVA, Ischemic, Acute. 


\section{Introducción}

La primera causa de muerte a nivel mundial son las Enfermedades Cardiovasculares (ECV). Se estima que cada año mueren más personas por ECV que por cualquier otra causa. En el año 2015 murieron por esta causa un aproximado de 17,7 millones de personas. Esta cifra representa un 31\% de todas las muertes registradas en todo el mundo. De este total, 7,4 millones fueron por causa de cardiopatía coronaria, y 6,7 millones, a los Accidentes Cerebrovasculares (ACV). (Organización Mundial de la Salud - OMS, 2017)

Un Accidente Cerebrovascular (ACV), también llamado ictus, es una verdadera emergencia médica. Existen dos tipos: el isquémico y el hemorrágico. El ACV o ictus isquémico es el tipo más común. Por lo general, se origina por un coágulo sanguíneo que bloquea o tapa un vaso sanguíneo en el cerebro. Este taponamiento impide que la sangre fluya hacia este órgano. En consecuencia, las células del cerebro comienzan a morir, en cuestión de minutos. Otra causa del ACV es la estenosis o estrechamiento arterial. Pueden originarse por la aterosclerosis. "Los ataques isquémicos transitorios se producen cuando la sangre no llega al cerebro por unos instantes". (Enciclopedia Médica ADAM, 2019)

En tal sentido, la terapia tromboembolítica consiste en el uso de fármacos que tienen la finalidad de romper o disolver los coágulos de sangre, que son la primera causa de Ios ACV. (Enciclopedia Médica ADAM, 2020)

"Estos medicamentos se pueden administrar por vía intravenosa (trombólisis endovenosa) o intraarterial (trombólisis intraarterial). En tal sentido, la trombólisis es la disolución de un coágulo sanguíneo". (Texas Heart Institute, s.f.)

En cuanto a las estadísticas Escudero et al. (2010) refieren que de $80-85 \%$ de los ictus son de tipo isquémico. La oclusión embó- lica a partir del corazón o desde lesiones arterioescleróticas de las arterias proximales, es la causa más frecuente. El ictus isquémico "es la tercera causa de muerte en los países desarrollados y la primera causa de discapacidad en adultos. La enfermedad provoca un efecto devastador sobre la calidad de vida del paciente y, además, supone un altísimo coste sanitario". (p. 371)

Con relación a las consecuencias y complicaciones, un ACV puede causar discapacidades temporales o permanentes, dependiendo del tiempo en que el cerebro carece de flujo sanguíneo y de qué parte resultó afectada. Algunas de las complicaciones pueden ser las siguientes:

- Parálisis o pérdida del movimiento muscular

- Dificultad para hablar o tragar

- Pérdida de memoria o dificultades para pensar

- Problemas emocionales

- Dolor

- Cambios en la conducta y en la capacidad de cuidado personal, entre otras. (Clínica Mayo, 2020)

Wardlaw et al. referido en Latour \& Galdos, (2012) destacan acerca de la trombólisis precoz en pacientes con ictus isquémico, que esta reduce el área de infarto cerebral, además de mejorar los resultados funcionales a los 3 meses. Asimismo, el beneficio del tratamiento disminuye de manera continua con el tiempo transcurrido desde el inicio de los síntomas, razón por la cual, debe practicarse lo antes posible. (p. 325)

Por otra parte, García et al. (2019) refieren que "es indispensable para el clínico estar actualizado en cuanto a los avances diagnósticos y terapéuticos que han demostrado impactar en términos de calidad de vida y funcionalidad en pacientes con ACV isquémico agudo". (p. 17) 
En consecuencia, el propósito fundamental del presente estudio es plasmar los aspectos más resaltantes relacionados con la trombólisis intraarterial como tratamiento de pacientes con ACV isquémico agudo.

\section{Materiales y métodos}

La presente investigación se desarrolló mediante una metodología de tipo documental o bibliográfica. Para lo cual se implementó una búsqueda de material bibliográfico digitalizado, que representó la base fundamental para el análisis y posterior compendio del tema de la trombólisis intraarterial como tratamiento de pacientes con ACV isquémico agudo.

Para la búsqueda se utilizaron diversas bases de datos y algunas páginas web del área de la salud con reconocimiento científico a nivel internacional. Entre las bases de datos utilizadas se pueden destacar: SciELO, Redalyc, Medigraphic, entre otros. Algunas de las páginas web utilizadas fueron: los portales de la Organización Mundial de la Salud (OMS), Texas Heart Institute, Clínica Mayo y Manuales MSD.

El tipo de material consistió en: informes, protocolos, tesis de grado y otras clases de contenidos. Fueron excluidos contenidos repetidos, las editoriales o cartas editoriales, anotaciones académicas y todo documento con bajo nivel de sustento científico o evidencia.

El tiempo de búsqueda se llevó a cabo durante el mes de noviembre del presente año. En cuanto a los descriptores, fueron usaron los siguientes: "trombólisis intraarterial", "terapia trombolítica"; "ACV isquémico agudo", "tratamiento del ACV isquémico agudo" y "trombólisis intraarterial en ACV isquémico agudo". La información localizada se fue filtrando bajo algunos criterios importantes tales como: idioma español, mayor relevancia y correlación temática. Igualmente, fueron seleccionados los trabajos de más actualidad (fecha de publicación de hace 10 años).
Cabe destacar que tanto la metodología como el análisis y la argumentación que se expusieron en la presente investigación se llevaron a cabo de forma consensuada entre todos los miembros del equipo investigador.

\section{Resultados}

La gran mayoría de los ACV isquémicos agudos, un $80 \%$ aproximadamente, se producen como consecuencia de un proceso oclusivo aterotrombótico o tromboembólico, en virtud de lo cual, la recanalización expeditiva de la arteria cerebral ocluida significaría la posibilidad de limitar la extensión de la lesión. En tal sentido, cabe resaltar que el uso de los trombolíticos para el tratamiento del ACV isquémico agudo se llevó a cabo a finales de los años 50. Asimismo, se llevaron a cabo muchos estudios con un reducido número de pacientes, en donde no se objetivaba ventajas por causa del incremento de complicaciones como hemorragias, en consecuencia, se suspendió su uso. No obstante, estudios clínicos y experimentales, por medio del uso de controles angiográficos, lograron demostrar que "la recanalización de oclusiones agudas en el territorio carotídeo y vertebro basilar pueden ser obtenidas con trombólisis, sobre todo cuando se administra en infusión intraarterial y dentro de las tres primeras horas del ACV isquémico agudo". Actualmente, se admite que la trombólisis es parte principal del tratamiento de emergencia de la mayoría de los pacientes con ictus isquémico, y que se dispone de un fármaco (t-PA) el cual consigue notoriamente mejorar el pronóstico y la recuperación de estos pacientes. (Carrizosa, Herrero López, \& Jareño Chaumela, 1999)

Las terapias de reperfusión en ACV isquémico agudo son:

Trombólisis intravenosa (IV).

Trombólisis intraarterial (IA).

Trombectomía mecánica. (García, y otros, 2019, p. 9)

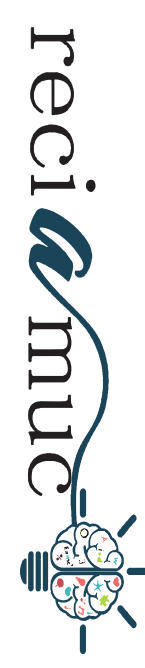


Siguiendo con la historia, más específicamente de la trombólisis intraarterial (IA), Mena (2013) refiere que esta se inició durante la década de los años 80 , con la trombólisis IA usando medicamentos como Urokinasa y tPA. Esta terapia se usó posterior al desarrollo de muy pequeños microcatéteres los cuales permitían ser navegados hasta los vasos intracerebrales por medio del uso de los métodos angiográficos y después inyectando localmente en el trombo vía IA el medicamento. En un principio, la trombólisis IA se indicaba en pacientes que presentaban clínicamente entre 3-6 horas del inicio de cuadro clínico o que tenían alguna contraindicación para la trombólisis sistémica. (p. 132)

En la actualidad, la trombólisis intraarterial bajo control angiográfico de un trombo o un émbolo, en ocasiones puede emplearse para los ACV mayores, si los síntomas comenzaron hace $<6$ horas, sobre todo en ACV por causa de oclusiones grandes de la arteria cerebral media y que no pueden ser tratadas con tPA recombinante IV. Los coágulos de la arteria basilar pueden ser lisados intraarterialmente hasta 12 horas después del inicio del proceso, en ocasiones puede ser más tarde dependiendo de las circunstancias clínicas. "Este tratamiento, aun cuando es el estándar de asistencia en algunos centros importantes para accidente cerebrovascular, muchas veces no está disponible en otros hospitales". (Giraldo, 2017)

\section{Indicaciones para recibir tratamiento en- dovascular (TEV) en ictus isquémico agu- do}

Según la Guía para el manejo del ictus isquémico agudo de la Sociedad Valenciana de Neurología, (2018) deberían recibir TEV, realizado preferentemente mediante stent recuperables, aquellos sujetos que cumplan todos los siguientes criterios (recomendación clase I, nivel evidencia A):

- Ictus isquémico que ha recibido tratamiento con rtpa IV dentro de las prime- ras 4,5 horas desde el inicio sin alcanzarse la recanalización.

- Independencia funcional previa medida mediante escala modificada de Rankin $=0-1$.

- Ictus secundario a oclusión de arteria carótida interna $(\mathrm{ACl})$ cervical o arteria cerebral media (ACM) segmento M1.

- Edad mayor o igual a 18 años.

- Puntuación NIHS mayor o igual a 6.

- Puntuación ASPECTS mayor o igual a 6.

- Inicio del tratamiento (punción arterial) dentro de las 6 primeras horas desde el inicio de los síntomas. (p. 10)

\section{Eficacia de la trombólisis intraarterial}

La eficacia y la seguridad del tratamiento trombolítico exclusivamente intraarterial se han constatado en diversos trabajos. El ensayo PROACT II referido por Escudero et al. (2010) en cuanto a la efectividad de este tratamiento expresó lo siguiente:

El ensayo PROACT II evaluó el tratamiento intraarterial con pro uroquinasas frente a placebo en el ictus por oclusión de la ACM dentro de las primeras $6 \mathrm{~h}$. En el grupo de tratamiento, las tasas de hemorragia intracraneal sintomática fueron del $10 \%$, con una recanalización del $66 \%$ (el $18 \%$ en el grupo control), a pesar de que el tiempo medio hasta el tratamiento fue de $5 \mathrm{~h}$ y media. A los 90 días, el $40 \%$ de los pacientes tratados con pro uroquinasa tenía una escala de Rankin inferior o igual a 2 frente a un $25 \%$ del grupo control, lo que demuestra la eficacia del tratamiento en términos de mejoría funcional. (p. 375)

Asimismo, estos autores en su estudio presentaron el caso de una mujer de 72 años con ictus isquémico agudo por obstrucción de la arteria basilar, grave y con rápido deterioro del nivel de conciencia, y tetraparesia la cual precisó intubación orotraqueal y ventilación mecánica. Se consiguió la reca- 
nalización completa tras el tratamiento con uroquinasa intraarterial e implantación del stent. El control realizado en la unidad de cuidados intensivos mediante sonografía Doppler transcraneal mostró la recanalización de la circulación posterior con permeabilidad completa de la arteria basilar (ver Figura 1). Asimismo, presentaron otros casos clínicos de ACV isquémico tratados con trombólisis intraarterial donde concluyeron lo siguiente:

En la actualidad, se acepta sin discusión como primera opción el tratamiento con rt-PA intravenoso, pero sus estrictos criterios de exclusión, la limitada ventana terapéutica de 3 h y los pobres resultados en casos de obstrucción de gran vaso hacen que el tratamiento trombolítico intraarterial asociado o no al tratamiento intravenoso pueda ser una gran alternativa como se ha demostrado en varios estudios. En nuestra serie, se obtuvieron unos buenos resultados funcionales con escasas complicaciones técnicas. (p. 376)
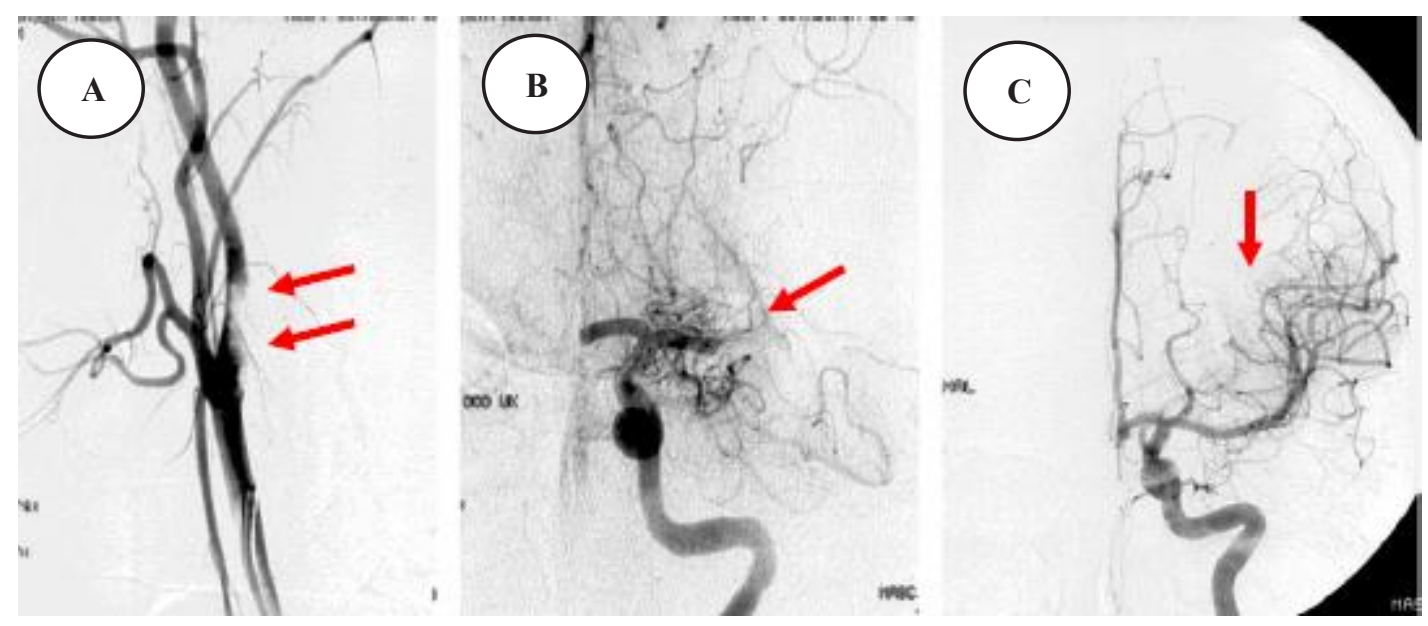

Figura 1. A) Arteriografía selectiva de la arteria carótida común derecha con gran defecto de repleción en el origen de la arteria carótida interna, que se corresponde con un trombo. B) Obstrucción asociada en el segmento M1 intracraneal de la arteria cerebral media. C) Control final tras la aspiración del trombo y la trombólisis farmacológica con 550.000 UI de uroquinasa. Recuperado de: "Tratamiento endovascular y trombólisis intraarterial en el ictus isquémico agudo". Escudero, D. et al. (2010). Medicina Intensiva. 34 (6). p. 376. Recuperado de: https://medintensiva.org/index.php?p=revista\&tipo=pdfsimple\&pii $=$ S02 10569110000367

\section{Limitaciones}

El problema que presenta el tratamiento intraarterial es que este necesita una infraestructura importante, es decir, personal médico que se encuentre altamente entrenado y especializado. La escasa disponibilidad de un servicio de neuro radiología intervencionista de guardia es una gran limitación para este tipo de tratamiento. En la actualidad, existe cierta discriminación negativa en el tratamiento del ictus agudo, con una evidente desventaja en infraestructura y recursos humanos en comparación con la enfermedad coronaria. Esto está confirmado en algunos estudios que demuestran que el tratamiento trombolítico intravenoso e intraarterial se usa, en el 1,4 y el 0,3\% de los pacientes, respectivamente. "Considerando que la enfermedad cerebrovascular constituye una de las primeras causas de mortalidad y discapacidad, su tratamiento multidisciplinario constituye, sin duda, uno de los desafíos más importantes para la sanidad en los próximos años". (Escudero et al., 2010, p. 378)

\section{Conclusiones}

La trombólisis, en líneas generales, es parte fundamental del tratamiento de emergencia de la mayoría de los pacientes con ACV isquémico agudo, cuya eficacia se encuentra comprobada científicamente, mejorando el 
pronóstico y la recuperación de estos pacientes.

La terapia trombolítica intraarterial en ACV isquémico agudo, conjuntamente o no, con la terapia IV es una gran alternativa, con excelentes resultados ampliamente comprobados. En virtud de lo cual, este tratamiento ha llegado a ser el estándar de asistencia en algunos centros importantes para ACV, mientras que, en muchos otros por razones técnicas o de falta de especialización, aún se encuentra ausente.

La misión fundamental en el tratamiento del ACV isquémico agudo es impedir las pérdidas de tiempo en su aplicación con la finalidad de evitar los daños y complicaciones que pueda acarrear esta enfermedad. No obstante, este tipo de tratamiento, por su requerimiento de alta especialización, se encuentra reservado en muchos países a centros con alta tecnología. En consecuencia, resulta imperioso invertir en la capacitación del personal de salud y generar un cambio radical en el estilo de manejo general de esta patología, que impacte en grandes áreas de atención para estos pacientes tales como equipos de emergencia, áreas de urgencias, neurología y $\mathrm{UCI}$, con la finalidad de lograr una atención precoz y mejorar el pronóstico y calidad de vida de estos pacientes. Más aún cuando se trata de una de las patologías más numerosas y con índices en ascenso a nivel mundial.

\section{Bibliografía}

Carrizosa, C., Herrero López, A., \& Jareño Chaumela, A. (1999). Fibrinolisis y fármacos trombolíticos. En F. e. Barranco Ruiz, Principios de Urgencias, Emergencias y Cuidados Críticos (Primera ed., pág. 1570). Uninet. Recuperado el 18 de Noviembre de 2020, de https://www.uninet.edu/tratado/ c060605.html

Clínica Mayo. (06 de Noviembre de 2020). Clínica Mayo. Recuperado el 10 de Noviembre de 2020, de https://www.mayoclinic.org/es-es/diseases-conditions/stroke/symptoms-causes/syc-20350113

Enciclopedia Médica ADAM. (03 de Septiembre de 2019). MedlinePlus. Recuperado el 03 de Noviem- bre de 2020, de https://medlineplus.gov/spanish/ ischemicstroke.html

Enciclopedia Médica ADAM. (07 de Julio de 2020). MedlinePlus. Recuperado el 02 de Noviembre de 2020, de https://medlineplus.gov/spanish/ency/article/007089.htm

Escudero, D., Molina, R., Viña, L., Rodríguez, P., Marques, P., Fernández, E., . . . Gilb, A. (2010). Tratamiento endovascular y trombólisis intraarterial en el ictus isque'mico agudo. Medicina Intensiva, 34(6), 370-378. doi:10.1016/j.medin.2010.01.010

García, C., Martínez, A., García, V., Ricaurte, A., Torres, I., \& Coral, J. (2019). Actualización en diagnóstico y tratamiento del ataque cerebrovascular isquémico agudo. Universitas Médica, 60(3), 1-17. doi:https://doi.org/10.11144/Javeriana.umed60-3. actu

Giraldo, E. A. (Marzo de 2017). Manuales MSD. Recuperado el 15 de Noviembre de 2020, de https:// www.msdmanuals.com/es/professional/trastornos-neurol\%C3\%B3gicos/accidente-cerebrovascular/accidente-cerebrovascular-isqu\%C3\%A9mi$\mathrm{co}$

Latour, J., \& Galdos, P. (2012). Trombolisis en el ictus isquémico agudo en centros sin unidad de ictus: ¿derivación a centro de referencia o tratamiento in situ? Medicina Intensiva, 36(5), 324-328. doi:10.1016/j.medin.2011.12.007

Mena, F. (2013). Tratamiento endovascular del Accidente Vascular Encefálico Agudo. Revista Médica Clínica Condes, 24(1), 131-138. Recuperado el 28 de Noviembre de 2020, de https://www.clinicalascondes.cl/Dev_CLC/media/Imagenes/PDF\%20revista\%20m\%C3\%A9dica/2013/1\%20enero/16-Dr. Mena.pdf

Organización Mundial de la Salud - OMS. (17 de Mayo de 2017). Organización Mundial de la Salud - OMS. Recuperado el 05 de Noviembre de 2020, de https://www.who.int/es/news-room/fact-sheets/ detail/cardiovascular-diseases-(cvds)

Pareja, A., Boscá, M., Galiano, R., Chamarro, R., Gil, R., Ponz, A., . . Domingo, F. (2018). Guía para el manejo del ictus isquémico agudo. Guía Clínica, Sociedad Valenciana de Neurología, Valencia. Recuperado el 21 de Noviembre de 2020, de https:// www.svneurologia.org/wordpress/wp-content/ uploads/2018/03/Gui\%CC\%81a-ictus-isquemico-agudo-2018.pdf

Texas Heart Institute. (s.f.). Texas Heart Institute. Recuperado el 18 de Noviembre de 2020, de https://www. texasheart.org/heart-health/heart-information-center/ topics/glosario-de-terminologia-cardiovascular/ 


\section{CITAR ESTE ARTICULO:}

Velez Bravo, M. D., Naranjo Apuntes, D. I., Méndez Barbosa, E. A., \& Barros Cabrera, J. A. (2020). Trombólisis intraarterial en ACV isquémico agudo. RECIAMUC, 4 (4 (esp), 14-21. https://doi.org/10.26820/reciamuc/4.(4).diciembre.2020.14-21

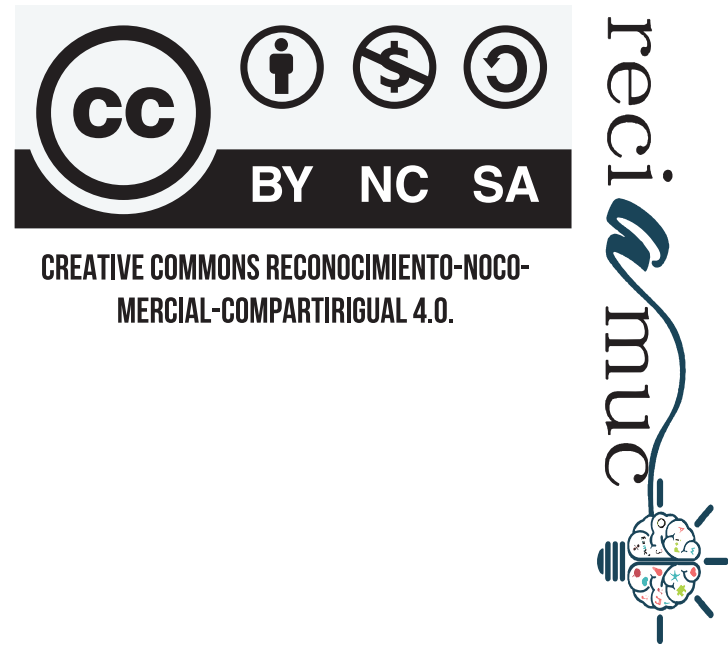

